\title{
Multi-responsive deep-ultraviolet emission in praseodymium-doped phosphors for microbial sterilization
}

\author{
Xinquan Zhou ${ }^{1}$, Jianwei Qiao ${ }^{1}$, Yifei Zhao ${ }^{1}$, Kai Han $^{1}$ and Zhiguo Xia ${ }^{1,2 *}$
}

\begin{abstract}
Perusing multimode luminescent materials capable of being activated by diverse excitation sources and realizing multi-responsive emission in a single system remains a challenge. Herein, we utilize a heterovalent substituting strategy to realize multimode deep-ultraviolet (UV) emission in the defect-rich host $\mathrm{Li}_{2} \mathrm{CaGeO}_{4}$ (LCGO). Specifically, the $\mathrm{Pr}^{3+}$ substitution in LCGO is beneficial to activating defect site reconstruction including the generation of cation defects and the decrease of oxygen vacancies. Regulation of different traps in LCGO:Pr ${ }^{3+}$ presents persistent luminescence and photostimulated luminescence in a synergetic fashion. Moreover, the up-conversion luminescence appears with the aid of the $4 f$ discrete energy levels of $\operatorname{Pr}^{3+}$ ions, wherein incident visible light is partially converted into germicidal deep-UV radiation. The multi-responsive character enables LCGO:Pr ${ }^{3+}$ to response to convenient light sources including $\mathrm{X}$-ray tube, standard UV lamps, blue and near-infrared lasers. Thus, a dual-mode optical conversion strategy for inactivating bacteria is fabricated, and this multi-responsive deep-UV emitter offers new insights into developing UV light sources for sterilization applications. Heterovalent substituting in trapmediated host lattice also provides a methodological basis for the construction of multi-mode luminescent materials.
\end{abstract}

Keywords: phosphor, deep ultraviolet, multi-mode luminescence, sterilization

\section{INTRODUCTION}

Growing attention has been drawn toward developing ultraviolet (UV) luminescent materials due to their great application prospects in the fields of sterilization, persistent tags, therapy, biomedicine, energy storage, and so on [1-6]. In particular, lanthanide-based UV luminescent materials represent an attractive alternative to design mercury-free solid-state UV light sources for sterilization in the current epidemic $[7,8]$. Since common UV light sources, including mercury lamps, light emitting diodes (LEDs), solid-state lasers, and excimer lamps, still suffer from some technical limitations such as environmental pollution (hydrargyrate), high cost, and short lifetime [9]. To develop new options, various inorganic UV phosphors were intensively investigated in the past few years, whereas studies on downshift luminescence are in the majority. Several recent studies turn to the UV long persistent luminescence
(PersL), upconversion luminescence (UCL) and up-conversion charging (UCC) PersL $[10,11]$. For example, Pan and co-workers $[3,12]$ developed new solar-blind UVC long persistent phosphors (LPP) and applied them to persistent tags. Yang et al. reported an X-ray-activated LPP $\mathrm{Cs}_{2} \mathrm{NaYF}_{6}: \mathrm{Pr}^{3+}[13]$ and a blue laser light-activated UC phosphor $\mathrm{Li}_{2} \mathrm{SrSiO}_{4}: \mathrm{Pr}^{3+}$ [14]. Both the above two phosphors were used for disinfection application. Nonetheless, there remains a challenge regarding the efficient integration of multi luminescence modes in a simple system.

Crucially, understanding the interactions among host lattice, luminescent centers and energy traps plays a significant role in realizing the coupling of complex emission modes. On the one hand, the inorganic host lattice can provide suitable crystallographic sites, crystal field and possible energy migration environment for the dopants, which directly contributes to the determination of luminescence features [15-17]. The natural defects and substitution defects of the host lattice, as well as the charge compensation effects will make for the generation and regulation of energy traps, thus contributing to the emergence of PersL and photo-stimulated luminescence (PSL) $[18,19]$. On the other hand, as preferred luminescence centers, lanthanide dopants with $4 f-4 f$ electronic transitions possess abundant absorption and emission energy levels, which enables the generation of photoluminescence (PL) and UCL [17,20,21]. Hence, coupling the interactions of host lattice, energy traps and luminescent centers provide the possibility of creating multimode luminescence in a synergetic fashion.

Herein, we present a rational design scheme for acquiring multimode UV emission in a single $\mathrm{Pr}^{3+}$-doped $\mathrm{Li}_{2} \mathrm{CaGeO}_{4}$ (LCGO) system, as shown in Fig. 1a. Trivalent praseodymium $\left(\mathrm{Pr}^{3+}\right)$ ion is selected as the UV emission centers in view of the fact that it possesses strong, broad, parity-allowed $4 f^{1} 5 d^{1} \rightarrow 4 f^{2}$ interconfigurational transitions [17,22]. The abundant and discrete $4 f$ energy levels of $\operatorname{Pr}^{3+}$ ions can act as intermediate states for UC process which thus can be employed as emitters in UC phosphors $[23,24]$. Whereas, the $4 f 5 d$ band energy within the range required for deep-UV emission is highly host-dependent due to that the $5 d$ electrons lie further from the nucleus and are not shielded from crystal field effects $[25,26]$. The occurrence of $\operatorname{Pr}^{3+} 4 f^{1} 5 d^{1} \rightarrow 4 f^{2}$ transitions in the deep-UV range requires a suitable crystal field environment to ensure a small stokes shift of less than about $0.37 \mathrm{eV}[27,28]$. Moreover, the germanate compound LCGO is regarded as a prototypical model system for accommodating $\operatorname{Pr}^{3+}$ dopant and LCGO can supply highly

\footnotetext{
${ }^{1}$ The State Key Laboratory of Luminescent Materials and Devices, Guangdong Provincial Key Laboratory of Fiber Laser Materials and Applied Techniques, School of Materials Science and Technology, South China University of Technology, Guangzhou 510641, China

${ }^{2}$ School of Physics and Optoelectronics, South China University of Technology, Guangzhou 510641, China

* Corresponding author (email: xiazg@scut.edu.cn)
} 
coordinated and charge-imbalanced cation sites for $\mathrm{Pr}^{3+}$ ions, which is highly likely to form a suitable crystal field and thus contribute to the deep-UV emission from $4 f-5 d$ transitions. LCGO possesses lower melting points and high volatility [29], which is beneficial to generating intrinsic defects (such as lithium vacancy $\mathrm{V}_{\mathrm{Li}}$, oxygen vacancy $\mathrm{V}_{\mathrm{O}}$ ), as shown in Fig. 1a, during the high-temperature treatment. Energy traps can be formed from these defects for storing and releasing excitation energies, further generating PersL and PSL [30,31].

By validating the above scheme, we demonstrate that $\operatorname{Pr}^{3+}$ doping in LCGO is able to reconstruct the defect sites, which is manifested as the formation of $\operatorname{Pr}_{\mathrm{Ca}}$ substitution defect and the reduction of oxygen vacancies. Theoretical calculations explicitly identify the regulation of trap types and distributions. Meanwhile, this heterovalent substitution strategy contributes to the creation of photochargeable energy traps that participate in the deep-UV delayed emission of $\mathrm{Pr}^{3+}$ under thermo- and photostimuli processes. Benefiting from the establishment of emission and trap centers simultaneously in the defect-rich host LCGO, the as-prepared LCGO: $\mathrm{Pr}^{3+}$ integrates multiple optical phenomena, namely PL, PersL, UCL and PSL. As expected, the resulting phosphor shows performance with strong deep-UV emission at $230-330 \mathrm{~nm}$, which is overlapping well with the germicidal effectiveness curve and can simultaneously respond to X-ray, UV, blue and near-infrared lasers. By utilizing the multi-stimuli responsive property of LCGO: $\mathrm{Pr}^{3+}$, we demonstrate a dual-mode optical conversion strategy for sterilization application. It shows that the deep-UV luminescence of this phosphor is sufficient to inactivate Staphylococcus (S.) aureus. Note that this phosphor can be charged repeatedly by X-ray, UV lamps or activated by blue laser feasibility, thus offering an attractive prospect for practical sterilization applications. Such a heterovalent substituting design strategy in the trap-mediated host lattice provides a methodological basis for the construction of multi-mode luminescent materials.

\section{EXPERIMENTAL SECTION}

\section{Synthesis}

Phosphors with stoichiometric molar compositions of $\mathrm{Li}_{2} \mathrm{Ca}_{1-x} \mathrm{Pr}_{x} \mathrm{GeO}_{4}(x=0.002-0.03)$ were synthesized using a solidstate method. Stoichiometric amounts of $\mathrm{Li}_{2} \mathrm{CO}_{3}$ (analytical reagent (AR)), $\mathrm{CaCO}_{3}$ (AR), $\mathrm{GeO}_{2}$ (AR), and $\mathrm{Pr}_{6} \mathrm{O}_{11}$ (99.99\%) were mixed and ground in an agate mortar for half an hour. The mixtures were heated at $850^{\circ} \mathrm{C}$ for $4 \mathrm{~h}$ in an air furnace and then cooled to room temperature (RT) in the furnace. Then the obtained samples were reground into fine powders for further characterization. The preformed discs were pressed using a pressing machine and then sintered at $850^{\circ} \mathrm{C}$ in air for $4 \mathrm{~h}$ to form solid ceramic discs.

\section{Characterizations}

The powder X-ray diffraction (XRD) patterns of $\mathrm{Li}_{2} \mathrm{Ca}_{1-x} \mathrm{Pr}_{x} \mathrm{GeO}_{4}$ were collected at $\mathrm{RT}$ by a Bruker D8 ADVANCE powder diffractometer with a linear VANTEC detector, operating at $40 \mathrm{kV}$ and $40 \mathrm{~mA}$ with monochromatized
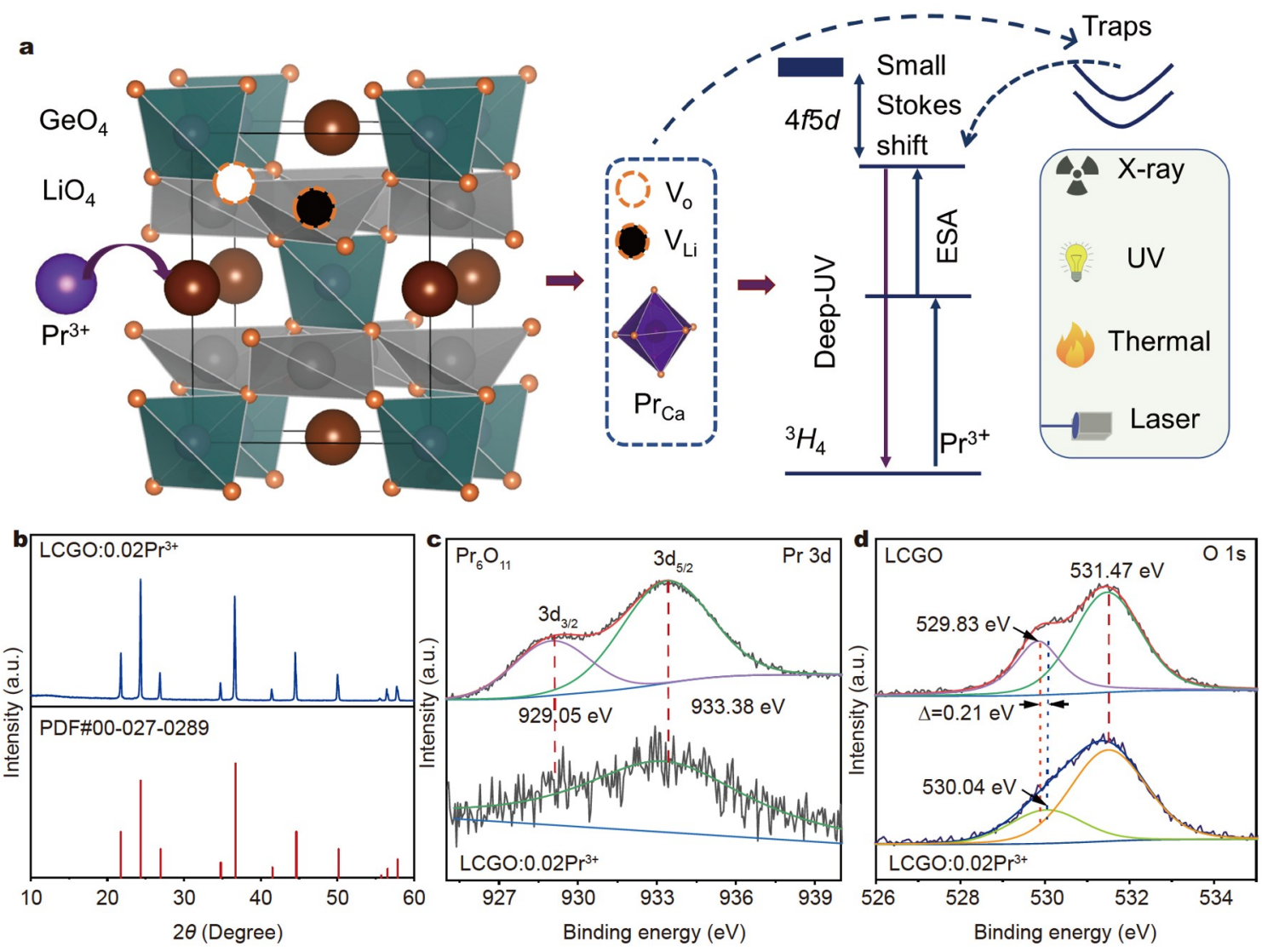

Figure 1 (a) Schematic illustration of designing functional particles with multi-responsive deep-UV emission in $\mathrm{Pr}^{3+}$-doped trap-rich host LCGO. (b) XRD pattern of LCGO:0.02 $\mathrm{Pr}^{3+}$ phosphor compared with the standard data of LCGO (\#PDF 00-027-0289). (c) XPS spectra of the LCGO:0.02Pr ${ }^{3+}$ sample and Pr $\mathrm{O}_{11}$ reference. (d) XPS analysis of O 1s for the representative LCGO and LCGO:0.02 $\mathrm{Pr}^{3+}$ samples. All XPS spectra were fitted after a Shirley background correction. 
$\mathrm{Cu} \mathrm{Ka}$ radiation $(\lambda=1.5406 \AA)$. X-ray photoelectron spectroscopy (XPS) was performed on an ESCALAB 250Xi spectrometer. RT PL spectra, PL excitation (PLE) spectra, PersL spectra, and decay curves were measured using a high-resolution spectrofluorometer (FLS1000, Edinburgh Instruments, UK) equipped with a Xe lamp as an excitation source and R928 photomultiplier (200-900 nm) as a detector. PersL spectra and PersL decay cures were measured after a UV lamp (25 W)/X-ray tube $(10 \mathrm{~W})$ excitation. The UCL spectra at RT were recorded by a Hitachi fluorescence spectrophotometer (F-4600) equipped with an external power tunable $450 \mathrm{~nm}$ laser diode array as excitation sources. Thermoluminescence (TL) glow curves measurements were performed with a TL dosimeter (FJ-427A1), with a heating rate of $2^{\circ} \mathrm{C} \mathrm{s}^{-1}$ from $\mathrm{RT}$ to $350^{\circ} \mathrm{C}$. The sample was irradiated for $5 \mathrm{~min}$ at RT, and then it was left for $3 \mathrm{~min}$ before the TL measurement. Moreover, an automated TL/optically stimulated luminescence reader (Ris $\varphi$ TL/OSL Da-20, DTU Nutech, Denmark) coupled with a spectrometer $(350-1000 \mathrm{~nm})$ (QE65 Pro, Ocean Optics) was used for PSL and TL twodimensional (2D) plot measurements. Before the PSL and TL 2D plot measurements, the sample was irradiated for $5 \mathrm{~min}$ at RT. Thermal images were recorded by a thermal infrared camera.

\section{Bacterial culture}

All utensils were autoclaved at $121^{\circ} \mathrm{C}$ for $20 \mathrm{~min}$ to ensure sterile S. aureus (ATCC 25923) were cultured in Luria-Bertani (LB) medium with shaking $\left(180 \mathrm{rmin}^{-1}\right)$ at $37^{\circ} \mathrm{C}$ and harvested at each exponential growth phase by centrifugation. The supernatant was discarded and the bacteria were resuspended in phosphate buffer saline (PBS). The concentration of bacteria was determined by measuring the optical density at $600 \mathrm{~nm}$ (OD600) via UV-vis spectroscopy. Bacterial cell suspensions were diluted to obtain cell samples containing $10^{6}$ to $10^{7} \mathrm{CFU} \mathrm{mL} \mathrm{m}^{-1}$.

\section{Plate counting method}

Phosphor sheets were covered on the 96-well plates containing bacteria solutions. The blue laser was used to induce the deepUV emission through the well plate for inactivating bacteria. Finally, $20 \mathrm{~mL}$ of culture medium in each well was plated on LB agar plates, and placed in an incubator at $37^{\circ} \mathrm{C}$ for $24 \mathrm{~h}$.

\section{Computational methods}

Theoretical simulations were carried out based on the density functional theory (DFT) and the generalized gradient approximation (GGA)-Perdew-Burke-Ernzerhof (PBE) exchangecorrelation functional for describing the interactions. The spinpolarized magnetic was considered in each calculation. The planewave cutoff energy was set to be $500 \mathrm{eV} .3 \times 3 \times 3 \mathrm{k}$-meshes centered at gamma point were used for the calculations of both structural optimization and electronic properties. All the structures were allowed to relax until the energy on the atoms was less than $1.0 \times 10^{-5} \mathrm{eV}$ and all the forces on atoms were below $0.1 \mathrm{eV} \AA^{-1}$

The defect formation energy was calculated by the following equation:

$$
\begin{aligned}
E^{f}(\text { defect })= & E_{\text {tot }}(\text { defect })-E_{\text {tot }}(\text { pristine })-\sum_{i} n_{i} \mu_{i} \\
& +q\left(\Delta E_{\text {fermi }}+E_{\mathrm{VBM}}\right)+E_{\text {error }},
\end{aligned}
$$

where $E_{\text {tot }}($ defect $)$ and $E_{\text {tot }}$ (pristine) are the total energy of the cells with and without defect. $\sum_{i} n_{i} \mu_{i}$ is the change in chemical potential before and after introducing the defects, in which $\mu_{i}$ is the corresponding chemical potential of the elements, and $n_{i}$ is the number of atoms. $\mu_{\mathrm{Pr}}$ and $\mu_{\mathrm{Ca}}$ were derived from $\operatorname{Pr}\left(\mathrm{P}_{3} /\right.$ $m m c)$ and $\mathrm{Ca}(F m \overline{3} m)$, respectively. $\mu_{\mathrm{O}}$ was estimated from the total energy of an $\mathrm{O}_{2}$ molecular placed in a cubic lattice of $10 \AA$ in dimensions. $q$ stands for the charge state of the defect, $\Delta E_{\text {fermi }}$ is the variation in the Fermi level before and after defect formation, and $E_{\mathrm{VBM}}$ is the valance band maximum.

\section{RESULTS AND DISCUSSION}

\section{Structural characterizations and DFT calculations}

LCGO and $\mathrm{Pr}^{3+}$-doped LCGO were prepared by a conventional solid state method, in which $\operatorname{Pr}^{3+}$ ions would substitute $\mathrm{Ca}^{2+}$ site with the general chemical formula $\mathrm{Li}_{2} \mathrm{Ca}_{1-x} \mathrm{Pr}_{x} \mathrm{GeO}_{4+\delta}(x=0-$ 0.03), (denoted as LCGO: $\left.x \operatorname{Pr}^{3+}\right), x$ is the doping amount of $\mathrm{Pr}^{3+}$ ions and $\delta$ is the variation of $\mathrm{O}$ amount relative to the original value. LCGO crystal belongs to the space group of $I 2 \mathrm{~m}$ with a body-centered tetragonal unit cell [32], which is composed of $\mathrm{CaO}_{8}$ dodecahedra and $\mathrm{SiO}_{4}$ or $\mathrm{GeO}_{4}$ tetrahedra linked by sharing edges, whereas the lithium atoms are in distorted $\mathrm{LiO}_{4}$ tetrahedra joined at the corners as displayed in Fig. 1a. $\operatorname{Pr}_{\mathrm{Ca}}$ occupation can be formed due to that the radius of $\mathrm{Pr}^{3+}$ ion is too big to occupy $\mathrm{LiO}_{4}$ or $\mathrm{GeO}_{4}$ tetrahedra. Such an occupation is highly coordinated to create a suitable crystal field for the occurrence of efficient $\operatorname{Pr}^{3+} 4 f^{1} 5 d^{1} \rightarrow 4 f^{2}$ interconfigurational transition for deep-UV emission [33], as disscussed latter. The XRD patterns (Fig. 1b) of the as-synthesized LCGO:0.02 $\mathrm{Pr}^{3+}$ phosphor matched well with the standard card of the LCGO crystal (PDF\#00-027-0289), and no other impurities were found, highlighting that $\mathrm{Pr}^{3+}$ only existed in substitutional form. When the $\mathrm{Pr}^{3+}$ ion was introduced into the LCGO host lattice, charge compensation needs to be considered due to the different valences of $\mathrm{Pr}^{3+}$ and $\mathrm{Ca}^{2+}$ ions. To clarify unambiguously the oxidation state of $\mathrm{Pr}^{3+}$ ion in our investigated compound and reveal the possible charge compensation mechanisms, XPS measurements were conducted on LCGO and LCGO:0.02 $\mathrm{Pr}^{3+}$ samples as well as the reference raw material $\operatorname{Pr}_{6} \mathrm{O}_{11}$, as shown in Fig. 1c, d. Only the Pr $3 d_{5 / 2}$ peak [34] at the binding energy of $933.38 \mathrm{eV}$ appears in the XPS spectrum of LCGO:0.02Pr ${ }^{3+}$, indicating that the non-equivalent doping has an effect on the electronic structure of the ions in the host lattice. For the $\mathrm{O} 1 \mathrm{~s}$ spectrum (Fig. 1d), it can be fitted into two peaks, which correspond to $\mathrm{V}_{\mathrm{O}}$ (peak at the low energy) and the lattice $\mathrm{O}$ (peak at the high energy) [35]. Moreover, the $V_{O}$ peak shifted to higher binding energies and its intensity decreased as $\mathrm{Pr}^{3+}$ was introduced into the host lattice, indicating that the number of $\mathrm{V}_{\mathrm{O}}$ decreased. This is reasonable because the positively charged $\mathrm{Pr}_{\mathrm{Ca}}$ will cause the reduction of positively charged $\mathrm{V}_{\mathrm{O}}$ to maintain the charge balance of the system. To clarify the critical role of $\mathrm{Pr}$ substitution, the DFT calculations were performed to further investigate the effects of $\operatorname{Pr}^{3+}$ doping on structural defects in the LCGO: $x \operatorname{Pr}^{3+}$ system.

To simplify the discussion, we primarily focused on the effects of $\operatorname{Pr}^{3+}$ on $\mathrm{V}_{\mathrm{O}}$ and the electronic structure of LCGO. Fig. 2a-c show the comparison of the projected density of states (PDOS) for the pristine and defective LCGO as well as $\mathrm{Pr}^{3+}$-doped defective LCGO. The creation of one $\mathrm{V}_{\mathrm{O}}$ introduces an in-gap state in LCGO, staying away from the conduction band minimum (CBM) (Fig. 2b), which may be related to energy traps. By contrast, the calculated DOS of the $\mathrm{V}_{\mathrm{O}}$-induced in-gap state 

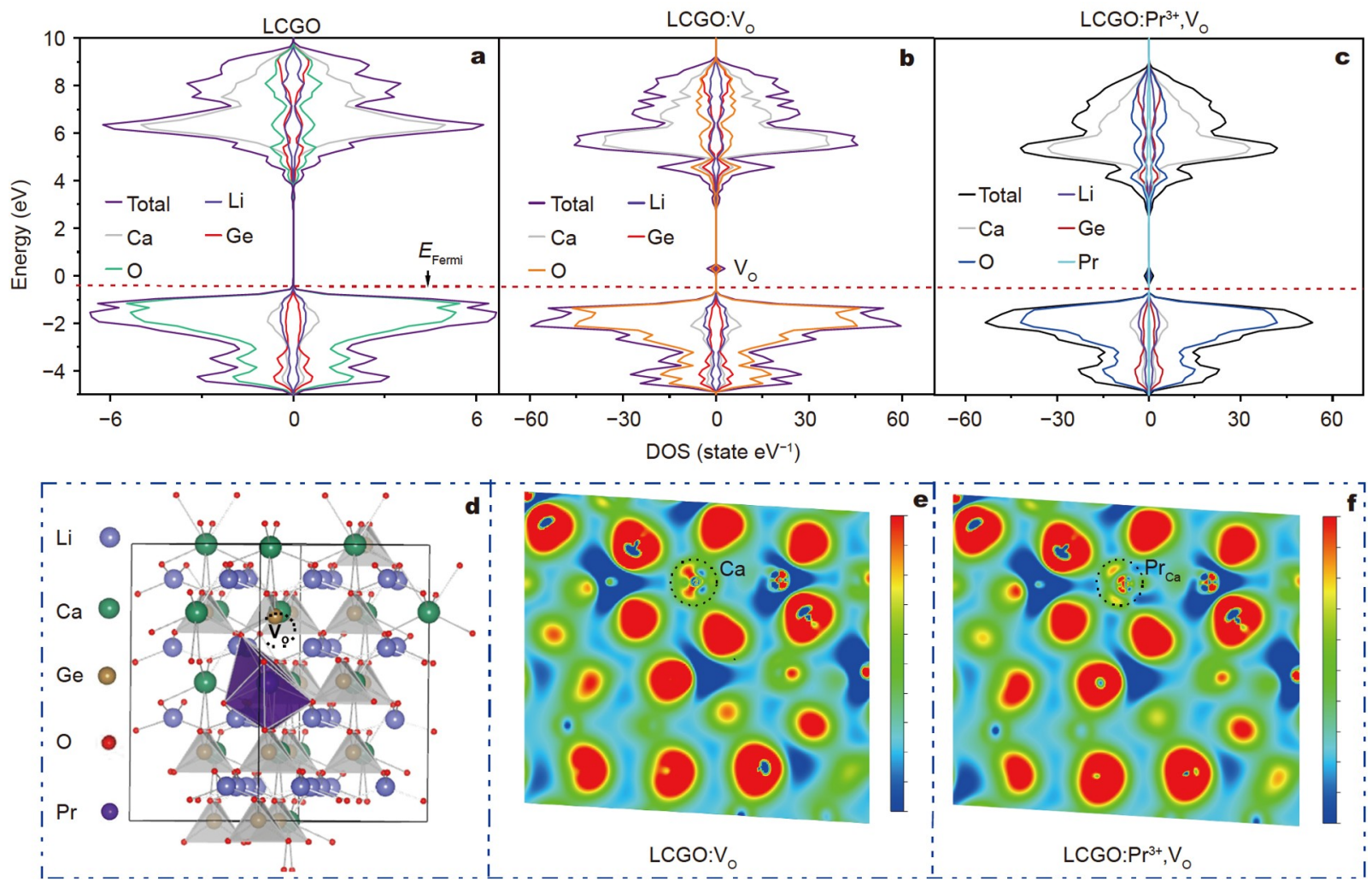

Figure 2 DOS of the pristine and defective LCGO. (a) DOS of the pristine LCGO. (b) DOS of LCGO: $\mathrm{V}_{\mathrm{O}}$. (c) DOS of LCGO:Pr ${ }^{3+}, \mathrm{V}_{\mathrm{O}}$. (d) The schematic shows the loss of one oxygen atomand doping Pr at Ca site in situ from LCGO host. The black circle represents the intentionally designed oxygen vacancies. $(\mathrm{e}, \mathrm{f})$ The ELF maps of LCGO: $\mathrm{V}_{\mathrm{O}}$ and LCGO: $\mathrm{Pr}^{3+}, \mathrm{V}_{\mathrm{O}}$.

decreased with introduced $\mathrm{Pr}^{3+}$ at $\mathrm{Ca}$ site (Fig. 2c). In addition, the formation energy of the coexistence of $\operatorname{Pr}^{3+}$ and $V_{O}$ is calculated to be $21.89 \mathrm{eV}$, which is higher than that of $\mathrm{V}_{\mathrm{O}}$ with $10.65 \mathrm{eV}$, indicating that the formation of $\mathrm{V}_{\mathrm{O}}$ is reduced after the doping of $\mathrm{Pr}^{3+}$. The calculated results demonstrate that there is a significant effect on $\mathrm{V}_{\mathrm{O}}$ when doping $\mathrm{Pr}^{3+}$ ions in the LCGO host. Fig. $2 \mathrm{~d}$ demonstrates the projected crystal structure of the LCGO: $\operatorname{Pr}^{3+}, \mathrm{V}_{\mathrm{O}}$, where one oxygen vacancy is created at the apical site of the $\mathrm{GeO}_{4}$ tetrahedron. Then the electron localization function (ELF) maps around the $\mathrm{Pr}$ atom at Ca site with $\mathrm{V}_{\mathrm{O}}$ in Ge site were analyzed, as shown in Fig. 2e, f. Comparing the ELF maps of LCGO: $\mathrm{V}_{\mathrm{O}}$ and LCGO: $\mathrm{Pr}^{3+}, \mathrm{V}_{\mathrm{O}}$, the electron localization area around $\operatorname{Pr}_{\mathrm{Ca}}$ curtails as creating an adjacent $\mathrm{V}_{\mathrm{O}}$ in $\mathrm{GeO}_{4}$ tetrahedron. That means that fewer electrons concentrate around $\operatorname{Pr}_{\mathrm{Ca}}$ site; however, this is not conducive to compensating the unbalanced charge caused by the non-equivalent doping and thus renders the system unstable. Therefore, it is reasonable that Pr doping leads to the reduction of $\mathrm{V}_{\mathrm{O}}$ in the system.

\section{Luminescence characterization}

The optical performance of LCGO:Pr ${ }^{3+}$ phosphor was evaluated in detail through DFT calculation and experimental measurements. The calculated bandgap $\left(E_{\mathrm{g}}\right)$ for the LCGO host is $3.8 \mathrm{eV}$ (Fig. 3a). This result demonstrates that LCGO is a suitable carrier for accommodating $\operatorname{Pr}^{3+}$ ions and able to provide enough closely energy gap between the excitation level and the bottom of the $\mathrm{CB}$ for inducing PersL process [31,36]. As illustration in Fig. 3b, doping $\operatorname{Pr}^{3+}$ ions into LCGO host does not cause a change in the energy gap $\left(E_{\mathrm{g}}\right)$. By converting the experimental diffuse reflectance spectrum using the Kubelka-Munk function [37], the estimated $E_{\mathrm{g}}$ value of LCGO:0.01 $\mathrm{Pr}^{3+}$ is $3.8 \mathrm{eV}$, which is consistent with the DFT calculation result. The PLE spectrum monitored at $254 \mathrm{~nm}$ emission consists of two broad excitation bands. One main band $(\sim 220-250 \mathrm{~nm})$ is located at about $245 \mathrm{~nm}$ and the other weak band $(\sim 200-220 \mathrm{~nm})$ is located at about $214 \mathrm{~nm}$, which correspond to the transitions of $5 d$ crystalfield component of $\operatorname{Pr}^{3+}$ [38]. Since $\operatorname{Pr}^{3+}$ ion is located at a highly symmetrical lattice site, it provides a suitable crystal field for efficient $4 f^{1} 5 d^{1} \rightarrow 4 f^{2}$ interconfigurational transition of $\mathrm{Pr}^{3+}$ with deep-UV emission. In response to $240-\mathrm{nm}$ light excitation, the LCGO: $\mathrm{Pr}^{3+}$ yields a broadband deep-UV emission ( $240-$ $330 \mathrm{~nm})$, that is overlapping well with the germicidal effectiveness curve ( 220-280 nm) [39] and phototherapy cure in broadband UVB emission $(290-320 \mathrm{~nm})$ [40].

Interestingly, LCGO:0.01 $\mathrm{Pr}^{3+}$ also possesses a strong absorption band around $450 \mathrm{~nm}$ corresponding to the ${ }^{3} \mathrm{H}_{4}{ }^{3} \mathrm{P}_{\mathrm{J}}$ transition of $\mathrm{Pr}^{3+}$, which matches well with the emission cross section of a convenient blue laser. Hence, under a 450-nm blue laser excitation, the LCGO:0.01 $\mathrm{Pr}^{3+}$ emits germicidal deep-UV UCL that is identical to PL. The UCL of this sample originates from the excited state absorption (ESA) process. Under 450-nm laser excitation, the blue photons are populated from the ground state level to the metastable intermediate state ${ }^{3} \mathrm{P}_{\mathrm{J}}$ and then absorbed to the lowest $4 f 5 d$ level as illustrated in Fig. $3 c[23,25]$. The slope of a linear regression fits on a double-logarithmic plot of integrated UC emission intensity and the laser power density gives a value of 1.93 (Fig. 3d), which is close to 2, certifying the twophoton UC process, as expected. 

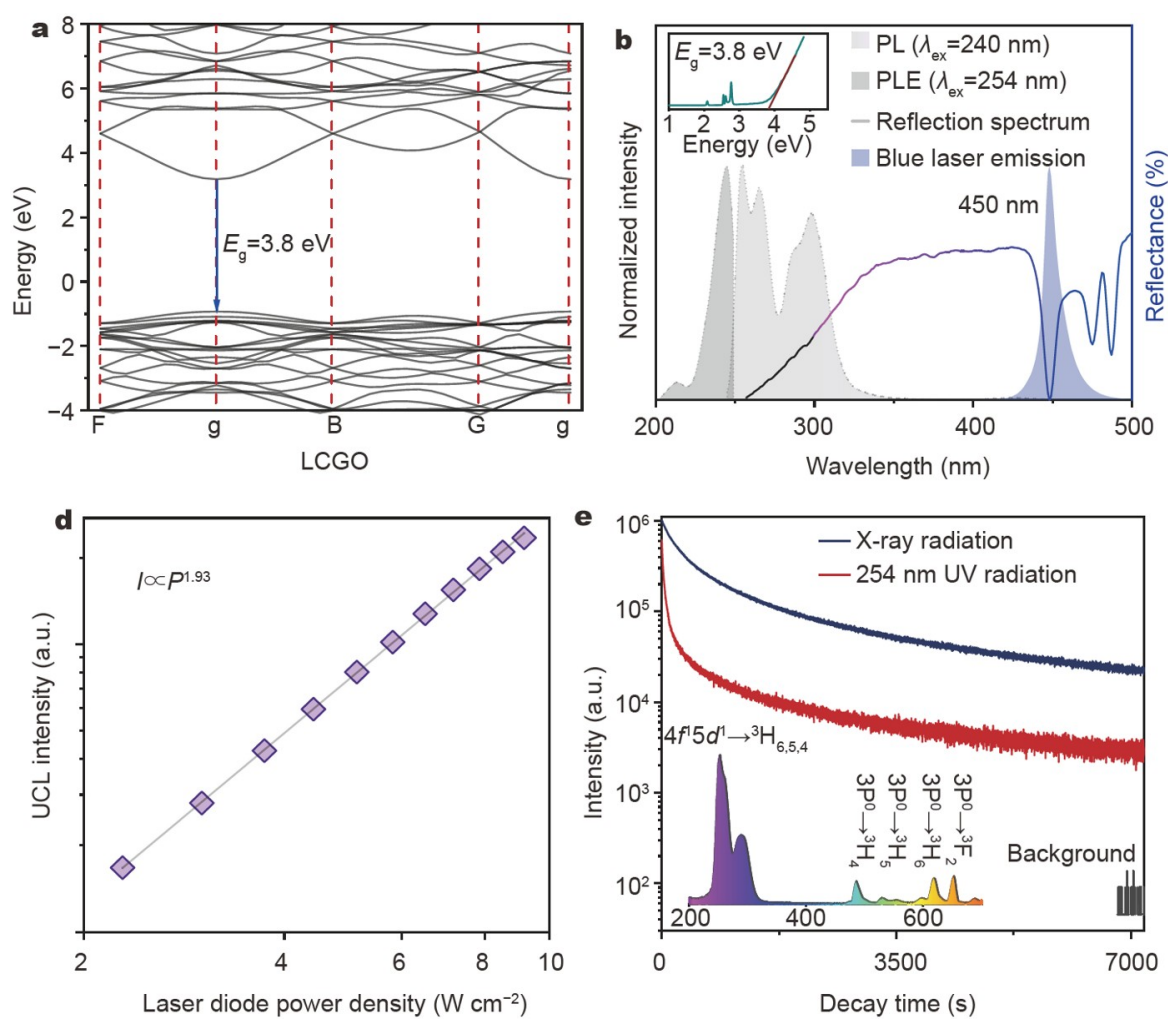

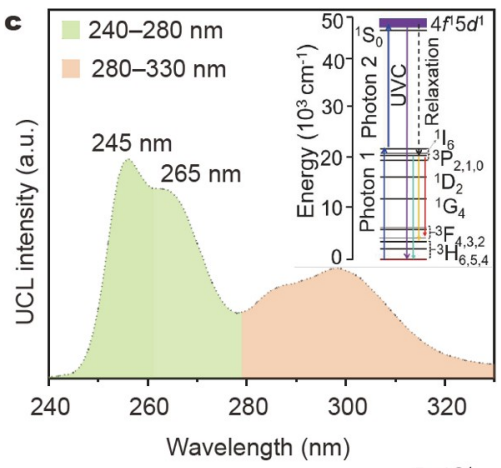

$\mathbf{f}$

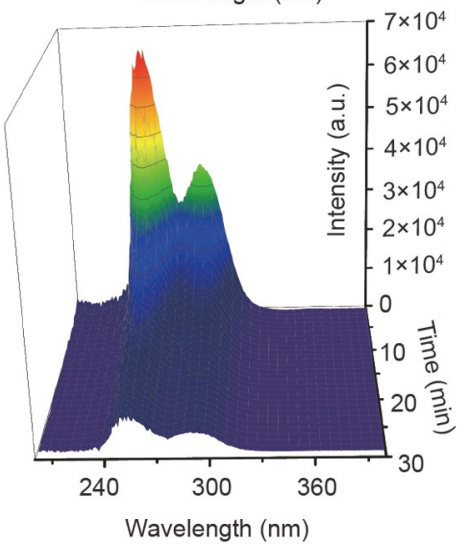

Figure 3 (a) DFT calculated electronic band structure of LCGO. (b) Normalized PLE, PL and diffuse reflectance spectra of LCGO:0.01Pr ${ }^{3+}$ at RT. The blue emission cross section is the emission of the laser used in this experiment. The inset shows the converted diffuse reflectance spectrum of the LCGO:0.01Pr ${ }^{3+}$ via Kubelka-Munk function $F(R)$ [37]. (c) UCL spectrum of LCGO:0.01 $\mathrm{Pr}^{3+}$ under blue laser excitation. The inset shows the energy level scheme of Pr ${ }^{3+}$ $4 f^{2} \rightarrow 4 f^{1} 5 d^{1}$ configuration and the possible UC mechanisms of $\operatorname{Pr}^{3+}$. (d) Illumination-power-density dependence of UCL intensity of LCGO:0.01Pr ${ }^{3+}$. Integrated UCL intensity $(I)$ versus the power density $(P)$ of the blue laser. (e) RT PersL decay curves of LCGO:0.006 $\mathrm{Pr}^{3+}$ after excitation by X-ray (10 W, blue line) and a UV low-pressure mercury lamp for $5 \mathrm{~min}$ as compared with the background signal. The bottom is the PersL spectrum of LCGO:0.006Pr ${ }^{3+}$. (f) The UV PersL spectra recorded at different times after UV irradiation was ceased.

The potential traps formed during the synthesis process and suitable $E_{\mathrm{g}}$ of LCGO:0.01Pr ${ }^{3+}$ suggest that this new deep-UV phosphor exhibits PersL phenomenon excepting the PL and UCL. When the excitation was ceased, deep-UV PersL emission accompanied by visible radiative transitions of $4 f^{2}-4 f^{2}$ can be obtained, as displayed in Fig. 3e. The PersL decay curves on LCGO:0.006 $\mathrm{Pr}^{3+}$ were recorded as a function of PersL intensity versus time and the recording lasted for $120 \mathrm{~min}$. After $120 \mathrm{~min}$ decay, the PersL intensity is still higher than the background signal. Notably, the sample charged by X-ray shows higher PersL intensity, over one order of magnitude stronger than that charged by UV. Fig. 3f shows the time-dependent PersL spectra recorded for $30 \mathrm{~min}$ after UV irradiation was ceased. The PersL intensity decreases quickly in the first few minutes and then decays slowly, which is consistent with the attenuation trend of the PersL decay curve.

\section{Analysis of trap regulation}

The traps in LPP can store and release excitation energy that forms the temperature dependency of PersL, namely TL. Herein, the TL was measured to analyze the properties of the traps and potential luminescence mechanisms of the LCGO phosphors. As depicted in the Fig. 4a, the LCGO and LCGO:0.006 $\mathrm{Pr}^{3+}$ possess two strong TL broadband peaks at 110 and $205^{\circ} \mathrm{C}$, representing the existence of shallow traps (STs) and deep traps (DTs), respectively. However, when $\operatorname{Pr}^{3+}$ ions are doped into the host, the proportion of STs increases obviously, indicating that the dopant $\mathrm{Pr}^{3+}$ has a significant effect on structural defects and thus affects the trap distribution, which also confirms the DFT results. Moreover, compared with X-ray, the UV light with relatively lower energy can fill more DTs. This particular phenomenon has also been reported in other trap-related LPPs, such as $\mathrm{ZnGa}_{2} \mathrm{O}_{4}: \mathrm{Cr}^{3+n}$ [41], $\mathrm{Li}_{5} \mathrm{Zn}_{8} \mathrm{Ga}_{5} \mathrm{Ge}_{9} \mathrm{O}_{36}: \mathrm{Cr}^{3+}, \mathrm{Ti}^{4+}$ [31] and $\mathrm{CaSnO}_{3}: \mathrm{Bi}^{2+}[42]$. The charge carriers can hardly escape from the DTs, leading a poor PersL intensity of the UV-charged sample, as compared in Fig. 3e. TL 2D mappings were carried out to clarify which PersL emission centers contribute to the TL glow curves, as shown in Fig. 4 b, c. It can be seen that the main TL glow peaks of the LCGO originate from intrinsic defects in the host. However, these traps are mainly distributed in deep regions $\left(150-300^{\circ} \mathrm{C}\right)$, which leads to thermal activation energy below/at $\mathrm{RT}$ and cannot release the captured charge carriers in traps, so that PersL is almost invisible at RT. The profile map in Fig. 4d shows that one of the main TL glow peaks of the LCGO: $0.006 \mathrm{Pr}^{3+}$ in shallow region $\left(50-150^{\circ} \mathrm{C}\right)$ is related to $\mathrm{Pr}^{3+}$ ions, indicating that $\mathrm{Pr}^{3+}$ acts as a role of energy traps in this host. Fig. $4 c$ does not show the deep-UV emission $(240-330 \mathrm{~nm})$ due to the limited detection range of spectrometer $(350-1000 \mathrm{~nm})$. Note that the PersL is RT TL, and hence the deep-UV emission in LCGO: $\operatorname{Pr}^{3+}$ contributes to TL peak. When the $\operatorname{Pr}^{3+}$ ions were introduced into the host, new defect $\operatorname{Pr}_{\mathrm{ca}}$ formed by heterovalent substitution, contributing to the increase of STs in the LCGO: 

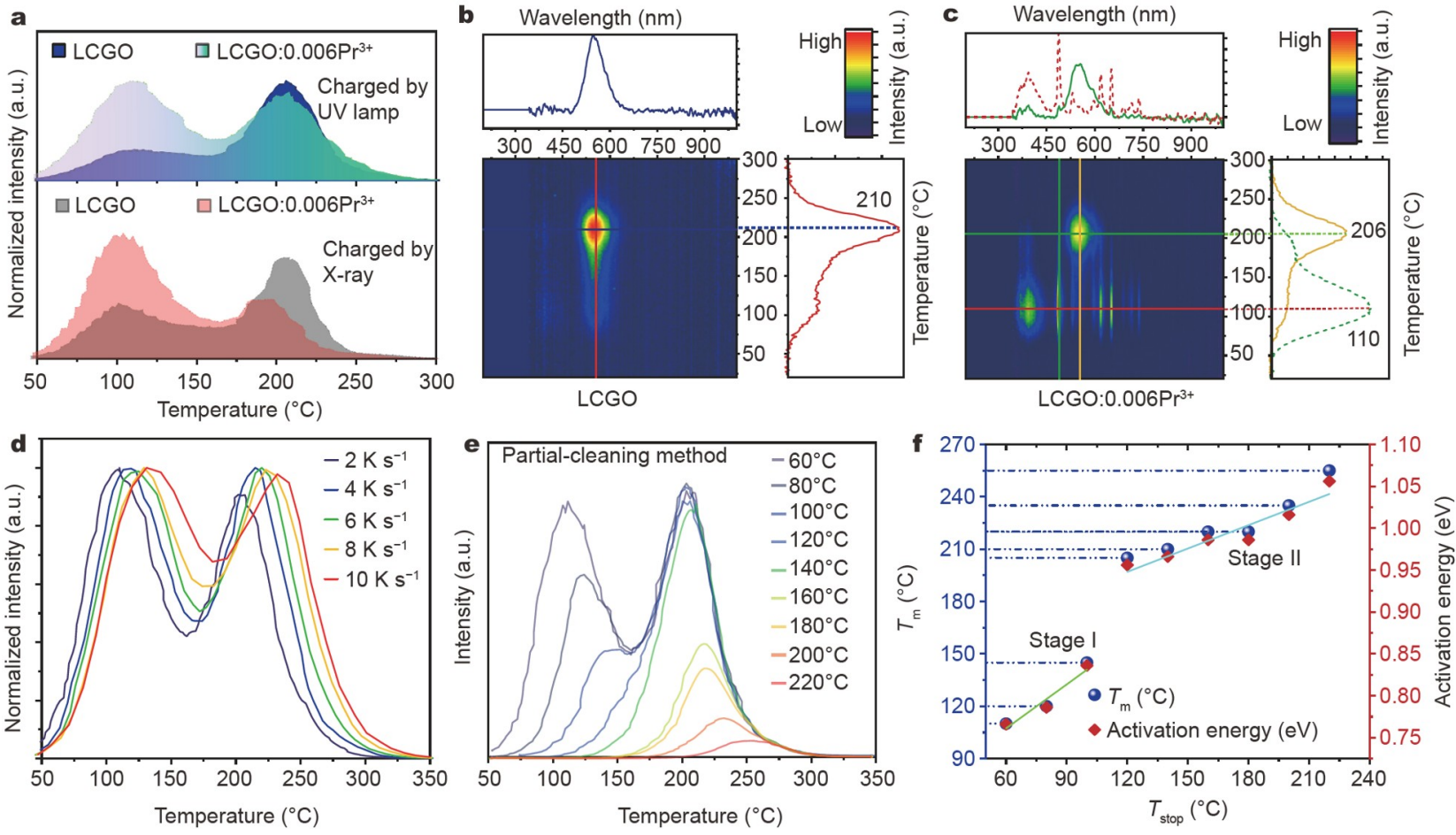

Figure 4 (a) The comparison of normalized TL glow curves of LCGO and LCGO:0.006 $\mathrm{Pr}^{3+}$ charged by UV and X-ray, respectively. (b, c) TL intensities of LCGO and LCGO:0.006 $\mathrm{Pr}^{3+}$ LPPs shown in wavelength-temperature contour plots. (d) Variation of the normalized TL glow curves with increasing heating rates. (e) Partial-cleaning method applied to determine the composition of the TL glow curves. (f) The comparison of $T_{\mathrm{m}}-T_{\text {stop }}$ diagram and the corresponding activation energies of LCGO:0.006 $\mathrm{Pr}^{3+}$.

$x \mathrm{Pr}^{3+}$. However, in order to satisfy the charge neutrality of the system, the number of $\mathrm{V}_{\mathrm{O}}$ decreased with the addition of $\mathrm{Pr}^{3+}$ ions, as shown in the TL glow curves in Fig. $4 \mathrm{~d}$, resulting in a reduction in the concentration of DTs. The result is also consistent with the DFT and XPS results analyzed earlier.

\section{Trapping and detrapping mechanism}

To clarify the trapping and detrapping mechanism of the LCGO: $x \mathrm{Pr}^{3+}$ samples, detailed TL measurements were carried out. Fig. $4 \mathrm{~d}$ shows the TL glow curves of LCGO:0.006 $\mathrm{Pr}^{3+}$ recorded as a function of increasing heating rate between 2 and $10 \mathrm{~K} \mathrm{~s}^{-1}$. As the heating rate increases, the temperature maximum $\left(T_{\mathrm{m}}\right)$ of the glow curve shifts toward higher temperatures. The reason can be explained mathematically [43]:

$\frac{\beta E}{k T_{\mathrm{m}}^{2}}=s \exp \left(\frac{-E}{k T_{\mathrm{m}}}\right)$,

where $\beta$ is the heating rate, $E$ is the activation energy, $k$ is Boltzmann constant, $8.617 \times 10^{-5} \mathrm{eV} \mathrm{K}^{-1}$ and $T_{\mathrm{m}}$ is the temperature maximum in kelvin $(\mathrm{K})$, and $s$ is the frequency factor. Hence, the $T_{\mathrm{m}}$ increases with the heating rate $\beta$. It is difficult to determine the activation energy for all of the traps and trap distribution in LCGO:0.006 $\mathrm{Pr}^{3+}$, since the TL glow curve is composed of several overlapping peaks. Therefore, a partialcleaning method was applied to determine the composition of a complex TL glow curve and the $E$ value of these traps, as shown in Fig. 4e, f. Such a method records the remaining TL glow curves after increasing the preheat temperature $\left(T_{\text {stop }}\right)$ in a step of $20 \mathrm{~K}$ (Fig. 4e), which can eliminate different fractions of the occupied traps depending on the excitation temperature [44]. As revealed in Fig. 4e, TL intensity gradually weakens with increasing $T_{\text {stop }}$ to $160^{\circ} \mathrm{C}$, whereas the TL intensity at $160-300^{\circ} \mathrm{C}$ remains constant. This result indicates that the electrons in the DTs are difficult to release without thermal activation. Meanwhile, the peak position of the TL curve shifts towards the higher temperature region with increasing $T_{\text {stop }}$, confirming the presence of a wide trap distribution in the investigated sample. An empirical way was applied to estimate the trap depths from the location of the TL peak [45]:

$E_{\mathrm{D}}=\frac{T_{\mathrm{m}}}{500}$,

where $E_{\mathrm{D}}$ is the average energy trap depth, and $T_{\mathrm{m}}(\mathrm{K})$ is the peak temperature. The dependence of evaluated trap depths on $T_{\text {stop }}$ is found distributed in two independent stage, $0.77-0.84 \mathrm{eV}$ (Stage I, belonging to STs), and $0.96-1.06 \mathrm{eV}$ (Stage II, belonging to DTs), as illustrated in Fig. 4f. By contrast, the trap depth in stage II increases with a smaller linear slope than stage I due to the slower de-trapping rates in this stage.

\section{Photostimulated persistent luminescence}

The co-existence of both quasi-continuous STs and DTs distributions is not only responsible for the unique PersL decays observed in the LCGO:Pr ${ }^{3+}$, but also for the PSL, as will be discussed in the follows. Fig. 5a shows the response of the UV pre-irradiated sample LCGO: $\mathrm{Pr}^{3+}$ after 5 min decay with $980 \mathrm{~nm} /$ $470 \mathrm{~nm}$ light stimulus. When the sample is stimulated by $980 \mathrm{~nm} / 470 \mathrm{~nm}$ light, charge carriers are released from DTs to STs and ready to recombine, and strong PSL can be observed immediately at that time. Once the stimulation is repealed, the PSL intensity drops suddenly to a low value due to that fewer carriers are released. Based on the experimental results and discussion presented above, we proposed a possible mechanism 

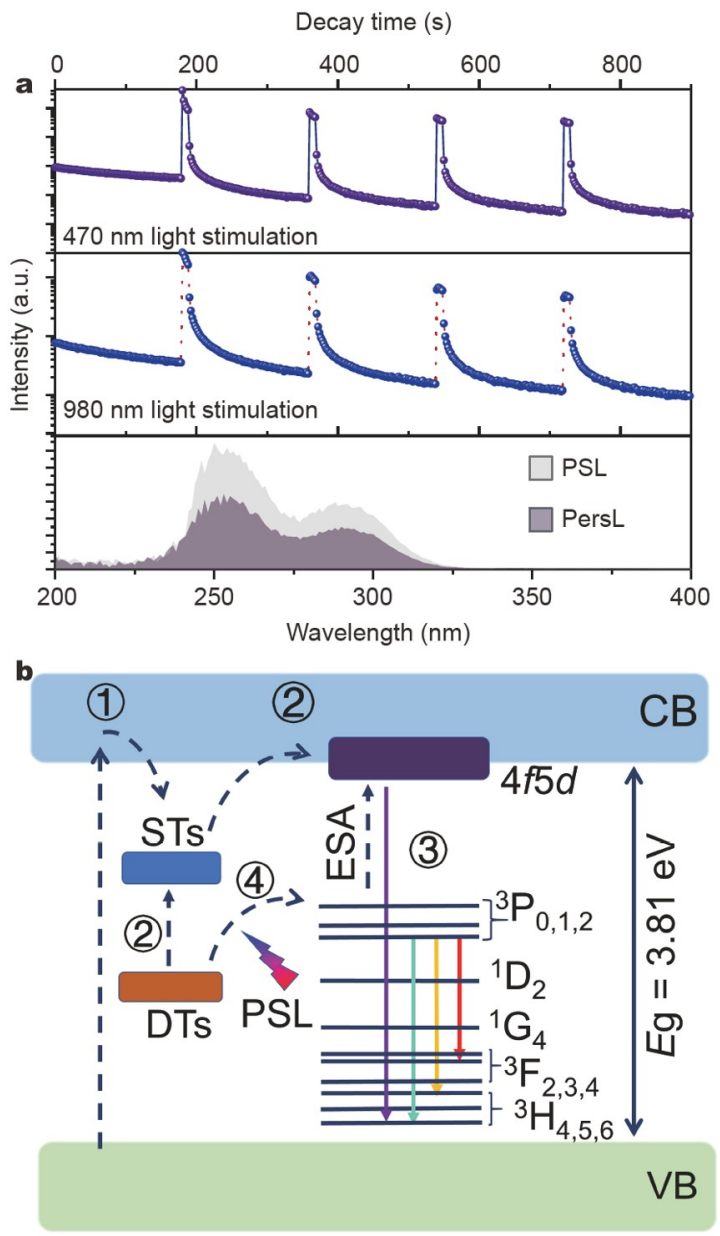

Figure 5 (a) Photo-stimulated decay curves and PSL of the charged LCGO: $\mathrm{Pr}^{3+}$ under periodical irradiation at 470 and $980 \mathrm{~nm}$. (b) The energy level diagram and proposed PersL and PSL mechanisms in LCGO:Pr ${ }^{3+}$.

to explain the multimode luminescence in this study, as shown in Fig. 5b. According to the DFT calculation, the band gap value is confirmed to be $3.8 \mathrm{eV}$. The electron traps formed by $\mathrm{V}_{\mathrm{O}}$ are far from the bottom of the $\mathrm{CB}$ and near the top of the valence band (VB). The $\mathrm{Pr}^{3+}$ energy levels are located in the middle of the forbidden band for illustration purposes. Upon UV irradiation, electrons are pumped into $\mathrm{CB}$ from $\mathrm{VB}$, leaving holes in the VB. At this point, the electrons in $\mathrm{CB}$ are captured by traps (process 1). After the excitation is ceased, some of trapped electrons are thermally detrapped into the CB from STs at RT. The STs and DTs are not absolutely independent so that the transition of electrons from DTs to STs is inevitable (process 2). Afterwards, the detrapped electrons in $\mathrm{CB}$ go down to the $5 d$ band of the $\mathrm{Pr}^{3+}$ then generating the excited $\mathrm{Pr}^{3+}$ ions, which subsequently gives rise to the PersL (process 3 ). The wide trap distribution and the low release rate of trapped electrons from DTs lead to weak but long lasting PersL. Under short-time stimulation with blue $(470 \mathrm{~nm})$ or near-infrared light $(980 \mathrm{~nm})$, some trapped electrons in DTs can be photo-released in two ways: reaching $\mathrm{CB}$ through STs (process 2) or tunneling to the ${ }^{3} \mathrm{P}_{\mathrm{J}}$ levels of $\mathrm{Pr}^{3+}$, and then an ESA process can possibly occur (process 4).

Bactericidal inactivation experiments

So far, we demonstrated the preparation of deep-UV phosphor
LCGO: $\mathrm{Pr}^{3+}$ with multimode luminescence and revealed that incorporating $\mathrm{Pr}^{3+}$ into the host lattice resulted in changing proportion of DTs and STs. The special trapping and detrapping processes suggest that this system shows individualized multimode luminescence. The broad features of deep-UV emission open up a diversity of potential applications. Herein, we design and demonstrate a bactericidal inactivation application that provides a new effective method for sterilization. As is well known, S. aureus is an important human pathogen that causes life-threatening infections and poses a serious public health threat [46]. As a proof of concept, the deep-UV phosphor developed here was used for killing $S$. aureus. In Fig. 6, we demonstrated the design and experimental results of sterilization with deep-UV UC and PersL emission. As shown in Fig. 6a, the LB agar plates are full of bacteria when plated with the untreated bacteria solutions and cultivated at $37^{\circ} \mathrm{C}$ for $24 \mathrm{~h}$. We covered the phosphor sheet above the transparent well plate containing bacteria solutions, and used blue laser to induce the deep-UV emission through the well plate for inactivating bacteria. The bacteria show different degrees of inactivation under different irradiation times (Fig. 6a).

All the bacteria were killed after $30 \mathrm{~min}$ of irradiation. In order to eliminate the interference of blue light and heat, more experiments were conducted. Fig. $6 \mathrm{~b}$ shows that the bacteria solutions exposed to blue laser (1 W) for $30 \mathrm{~min}$ on LB agar plates also grew full of bacteria. This means that the blue laser is not effective in sterilization. Besides, the blue laser treatment did not cause significant temperature changes around the well plate (Fig. 6d, e), which ruled out the effect of temperature on sterilization. The deep-UV PersL of the developed phosphor can also be used for killing $S$. aureus. The phosphor sheet was preirradiated by X-ray for $5 \mathrm{~min}$ and then kept close to the well plate containing bacteria solutions for $30 \mathrm{~min}$. After being cultured for $24 \mathrm{~h}$, the UV PersL-irradiated bacteria solutions produced significantly fewer bacteria than the untreated (Fig. 6c). The above results provide direct evidence that the UCL and PersL from our deep-UV phosphor can be used for sterilization. The readily available excitation sources highlight its enormous potential for practical applications.

\section{CONCLUSIONS}

A heterovalent substituting design strategy for developing $\mathrm{Pr}^{3+}$ doped LCGO deep-UV phosphor with multi-responsive character is reported, presenting excellent visible-to-UV conversion and energy storage capabilities. We demonstrated that the flexible response modes of LCGO: $\mathrm{Pr}^{3+}$ were derived from the crucial energy trap center reconstruction and abundant intermediate levels of $\mathrm{Pr}^{3+}$ in trap-mediated LCGO compound. DFT calculations verify the critical role of $\mathrm{Pr}^{3+}$ substitution on defectrelated traps: introducing the $\mathrm{Pr}^{3+}$ ion into host lattice contributes to the creation of $\mathrm{Pr}_{\mathrm{Ca}}$ defect and the decrease of $\mathrm{V}_{\mathrm{O}}$. It effectively regulated the proportion of DTs and STs, and then enabled the LCGO: $\mathrm{Pr}^{3+}$ to present PersL and PSL with deep-UV emission in a synergetic fashion. Upon 450-nm blue laser excitation, the phosphor also gives intense broad band deep-UV UCL that is overlapping well with the germicidal effectiveness curve. Hence, the deep-UV emission of LCGO:Pr ${ }^{3+}$ can be aroused by convenient light sources including X-ray tubes, laser diode arrays and UV lamps. Interestingly, the deep-UV UCL and PersL of this phosphor are sufficiently intense for sterilization. This study is expected to inspire the development of 


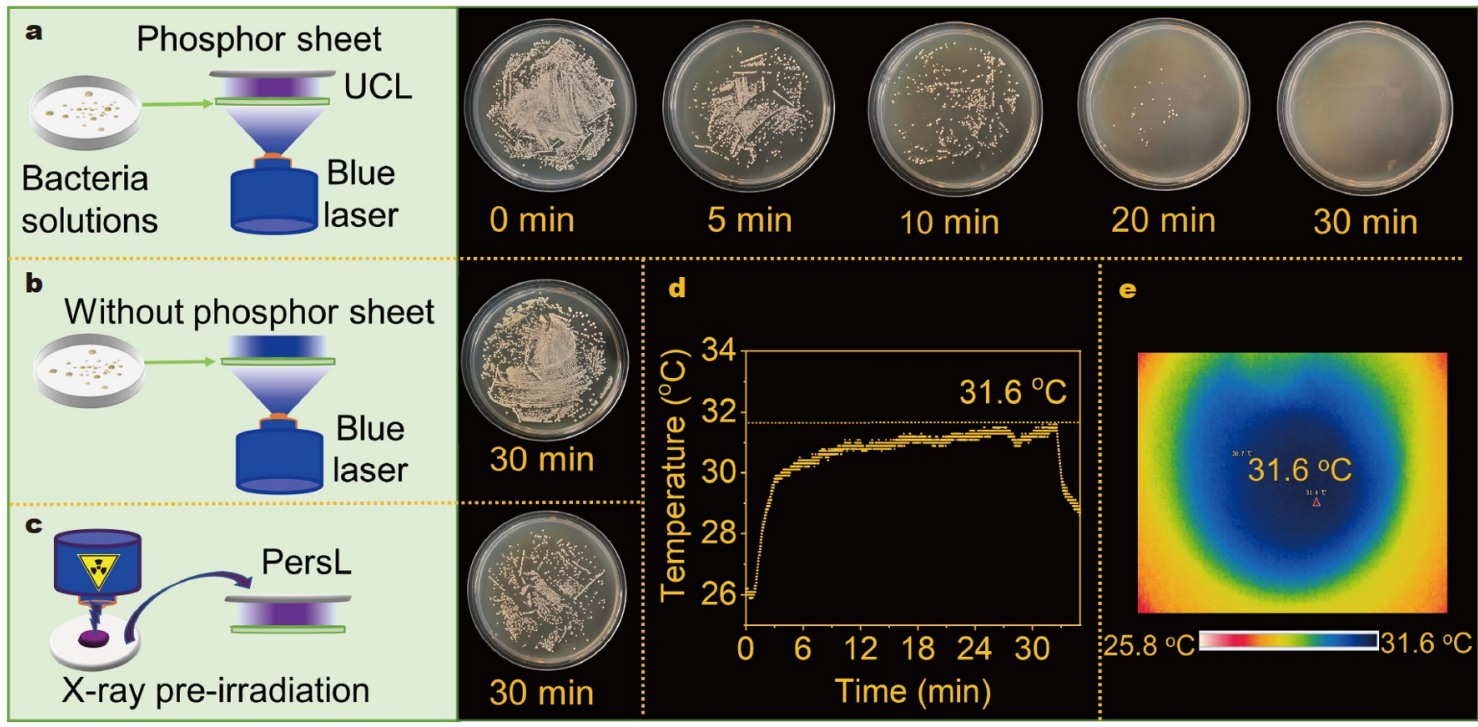

Figure 6 Design and experimental results of sterilization with deep-UV UC and PersL emission. (a) The 450-nm blue laser penetrates the well plates with bacteria solutions to illuminate the phosphor sheet and iduce the deep-UV emission to inactivate bacteria. (b) The result of culturing bacteria solutions after irradiation by a blue laser $(1 \mathrm{~W})$ for $30 \mathrm{~min}$. (c) Phosphor sheet was pre-irradiated by X-ray for $5 \mathrm{~min}$ and then used to inactivate $S$. aureus (ATCC 25923). $(d, e)$ Temperature curve and thermal image of the surrounding well plate with bacteria illuminated by blue light laser.

more excellent $\mathrm{Pr}^{3+}$-doped deep-UV phosphors with multiresponse for potential applications.

Received 17 August 2021; accepted 6 September 2021; published online 15 October 2021

1 Zhou X, Qiao J, Xia Z. Learning from mineral structures toward new luminescence materials for light-emitting diode applications. Chem Mater, 2021, 33: 1083-1098

2 Lyu T, Dorenbos P. Vacuum-referred binding energies of bismuth and lanthanide levels in $\operatorname{ARE}(\mathrm{Si}, \mathrm{Ge}) \mathrm{O}_{4}(\mathrm{~A}=\mathrm{Li}, \mathrm{Na} ; \mathrm{RE}=\mathrm{Y}, \mathrm{Lu})$ : Toward designing charge-carrier-trapping processes for energy storage. Chem Mater, 2020, 32: 1192-1209

3 Wang X, Chen Y, Liu F, et al. Solar-blind ultraviolet-C persistent luminescence phosphors. Nat Commun, 2020, 11: 2040

4 Song K, Mohseni M, Taghipour F. Application of ultraviolet lightemitting diodes (UV-LEDs) for water disinfection: A review. Water Res, 2016, 94: 341-349

5 Baron ED, Stevens SR. Phototherapy for cutaneous T-cell lymphoma. Dermatol Ther, 2003, 16: 303-310

6 Chen J, Loeb S, Kim JH. LED revolution: Fundamentals and prospects for UV disinfection applications. Environ Sci-Water Res Technol, 2017, 3: $188-202$

7 Sharma VK, Tan ST, Haiyang Z, et al. On-chip mercury-free deep-UV light-emitting sources with ultrahigh germicidal efficiency. Adv Opt Mater, 2021, 9: 2100072

8 Shining a light on COVID-19. Nat Photonics, 2020, 14: 337

9 Ronda C. Challenges in application of luminescent materials, a tutorial overview. Prog Electromagn Res, 2014, 147: 81-93

10 Yan S, Liu F, Zhang J, et al. Persistent emission of narrowband ultraviolet-B light upon blue-light illumination. Phys Rev Appl, 2020, 13: 044051

11 Yan $\mathrm{S}$, Gao Q, Zhao X, et al. Charging $\mathrm{Gd}_{3} \mathrm{Ga}_{5} \mathrm{O}_{12}: \mathrm{Pr}^{3+}$ persistent phosphor using blue lasers. J Lumin, 2020, 226: 117427

12 Yan S, Liang Y, Chen Y, et al. Ultraviolet-C persistent luminescence from the $\mathrm{Lu}_{2} \mathrm{SiO}_{5}: \mathrm{Pr}^{3+}$ persistent phosphor for solar-blind optical tagging. Dalton Trans, 2021, 50: 8457-8466

13 Yang YM, Li ZY, Zhang JY, et al. X-ray-activated long persistent phosphors featuring strong UVC afterglow emissions. Light Sci Appl, 2018, 7: 88
14 Yin Z, Yuan $\mathrm{P}$, Zhu Z, et al. $\mathrm{Pr}^{3+}$ doped $\mathrm{Li}_{2} \mathrm{SrSiO}_{4}$ : An efficient visibleultraviolet $\mathrm{C}$ up-conversion phosphor. Ceramics Int, 2021, 47: 48584863

15 Qiao J, Xia Z, Zhang Z, et al. Near UV-pumped yellow-emitting $\mathrm{Sr}_{9} \mathrm{MgLi}\left(\mathrm{PO}_{4}\right)_{7}: \mathrm{Eu}^{2+}$ phosphor for white-light LEDs. Sci China Mater, 2018, 61: 985-992

16 Wickleder MS. Inorganic lanthanide compounds with complex anions. Chem Rev, 2002, 102: 2011-2088

17 Qin X, Liu X, Huang W, et al. Lanthanide-activated phosphors based on $4 f-5 d$ optical transitions: Theoretical and experimental aspects. Chem Rev, 2017, 117: 4488-4527

18 Zhang JC, Pan C, Zhu YF, et al. Achieving thermo-mechano-optoresponsive bitemporal colorful luminescence via multiplexing of dual lanthanides in piezoelectric particles and its multidimensional anticounterfeiting. Adv Mater, 2018, 30: 1804644

19 Ren Y, Yang Z, Wang Y, et al. Reversible multiplexing for optical information recording, erasing, and reading-out in photochromic $\mathrm{BaMgSiO}_{4}: \mathrm{Bi}^{3+}$ luminescence ceramics. Sci China Mater, 2020, 63: $582-$ 592

20 Du Y, Ai X, Li Z, et al. Visible-to-ultraviolet light conversion: Materials and applications. Adv Photo Res, 2021, 2: 2000213

21 Du Y, Wang Y, Deng Z, et al. Blue-pumped deep ultraviolet lasing from lanthanide-doped $\mathrm{Lu}_{6} \mathrm{O}_{5} \mathrm{~F}_{8}$ upconversion nanocrystals. Adv Opt Mater, 2020, 8: 1900968

22 Tanner PA, Mak CSK, Faucher MD, et al. $4 f-5 d$ transitions of $\mathrm{Pr}^{3+}$ in elpasolite lattices. Phys Rev B, 2003, 67: 115102

$23 \mathrm{Hu} \mathrm{C}$, Sun C, Li J, et al. Visible-to-ultraviolet upconversion in $\mathrm{Pr}^{3+}$ : $\mathrm{Y}_{2} \mathrm{SiO}_{5}$ crystals. Chem Phys, 2006, 325: 563-566

24 Cates EL, Wilkinson AP, Kim JH. Delineating mechanisms of upconversion enhancement by $\mathrm{Li}^{+}$codoping in $\mathrm{Y}_{2} \mathrm{SiO}_{5}: \mathrm{Pr}^{3+}$. J Phys Chem C, 2012, 116: 12772-12778

25 Cates EL, Cho M, Kim JH. Converting visible light into UVC: Microbial inactivation by $\mathrm{Pr}^{3+}$-activated upconversion materials. Environ Sci Technol, 2011, 45: 3680-3686

26 Cates EL, Wilkinson AP, Kim JH. Visible-to-UVC upconversion efficiency and mechanisms of $\mathrm{Lu}_{7} \mathrm{O}_{6} \mathrm{~F}_{9}: \mathrm{Pr}^{3+}$ and $\mathrm{Y}_{2} \mathrm{SiO}_{5}: \mathrm{Pr}^{3+}$ ceramics. J Lumin, 2015, 160: 202-209

27 Dorenbos $\mathrm{P}$. The $5 d$ level positions of the trivalent lanthanides in inorganic compounds. J Lumin, 2000, 91: 155-176

28 Srivastava AM. Aspects of $\mathrm{Pr}^{3+}$ luminescence in solids. J Lumin, 2016, 169: $445-449$ 
29 Li Y, Gecevicius M, Qiu J. Long persistent phosphors-From fundamentals to applications. Chem Soc Rev, 2016, 45: 2090-2136

30 Li H, Liu Q, Ma J-, et al. Theory-guided defect tuning through topochemical reactions for accelerated discovery of UVC persistent phosphors. Adv Opt Mater, 2020, 8: 1901727

31 Zhou X, Ju G, Dai T, et al. $\mathrm{Li}_{5} \mathrm{Zn}_{8} \mathrm{Ga}_{5} \mathrm{Ge}_{9} \mathrm{O}_{36}: \mathrm{Cr}^{3+}, \mathrm{Ti}^{4+}$ : A long persistent phosphor excited in a wide spectral region from UV to red light for reproducible imaging through biological tissue. Chem Asian J, 2019, 14: 1506-1514

32 Gard JA, West AR. Preparation and crystal structure of $\mathrm{Li}_{2} \mathrm{CaSiO}_{4}$ and isostructural $\mathrm{Li}_{2} \mathrm{CaGeO}_{4}$. J Solid State Chem, 1973, 7: 422-427

33 Blasse G. Interaction between optical centers and their surroundings: An inorganic chemist's approach. Adv Inorg Chem, 1990, 35: 319-402

34 Ogasawara $\mathrm{H}$, Kotani A, Potze R, et al. Praseodymium $3 d$ - and $4 d$-core photoemission spectra of $\operatorname{Pr}_{2} \mathrm{O}_{3}$. Phys Rev B, 1991, 44: 5465-5469

35 Yang W, Li J, Liu B, et al. Multi-wavelength tailoring of a $\mathrm{ZnGa}_{2} \mathrm{O}_{4}$ nanosheet phosphor via defect engineering. Nanoscale, 2018, 10: 19039-19045

36 Ueda J, Leaño JL, Richard C, et al. Broadband near-infrared persistent luminescence of $\mathrm{Ba}\left[\mathrm{Mg}_{2} \mathrm{Al}_{2} \mathrm{~N}_{4}\right]$ with $\mathrm{Eu}^{2+}$ and $\mathrm{Tm}^{3+}$ after red light charging. J Mater Chem C, 2019, 7: 1705-1712

37 Simmons EL. Diffuse reflectance spectroscopy: A comparison of the theories. Appl Opt, 1975, 14: 1380-1386

38 van Pieterson L, Reid MF, Wegh RT, et al. $4 f^{n} \rightarrow 4 f^{n-1} 5 d$ transitions of the light lanthanides: Experiment and theory. Phys Rev B, 2002, 65: 045113

39 Kowalski W. UV Surface Disinfection. In: Kowalski W (ed.). Ultraviolet Germicidal Irradiation Handbook. Berlin, Heidelberg: Springer. 2009. 233-254.

40 Sasaki N, Yamashita T, Kasahara K, et al. UVB exposure prevents atherosclerosis by regulating immunoinflammatory responses. Arterioscler Thromb Vasc Biol, 2017, 37: 66-74

41 Maldiney $\mathrm{T}$, Bessière $\mathrm{A}$, Seguin $\mathrm{J}$, et al. The in vivo activation of persistent nanophosphors for optical imaging of vascularization, tumours and grafted cells. Nat Mater, 2014, 13: 418-426

42 Chen X, Li Y, Huang K, et al. Trap energy upconversion-like nearinfrared to near-infrared light rejuvenateable persistent luminescence. Adv Mater, 2021, 33: 2008722

43 Klasens HA, Garlick GFJ, Gibson AF. Discussion on "the electron trap mechanism of luminescence in sulphide and silicate phosphors". Proc Phys Soc, 1948, 61: 101-102

44 Van den Eeckhout K, Bos AJJ, Poelman D, et al. Revealing trap depth distributions in persistent phosphors. Phys Rev B, 2013, 87: 045126

45 Chen R. On the calculation of activation energies and frequency factors from glow curves. J Appl Phys, 1969, 40: 570-585

46 Chen F, Di H, Wang Y, et al. Small-molecule targeting of a diapophytoene desaturase inhibits $S$. aureus virulence. Nat Chem Biol, 2016, 12: $174-179$

Acknowledgements This work was supported by the National Natural Science Foundation of China (51961145101 and 51972118), the International Cooperation Project of National Key Research and Development Program of China (2021YFE0105700), Guangzhou Science \& Technology Project (202007020005), and the Local Innovative and Research Teams Project of Guangdong Pearl River Talents Program (2017BT01X137). The authors also thank Jiarong Liang and Prof. Bingfu Lei at South China Agricultural University for assistance with bactericidal inactivation.

Author contributions Zhou X performed the experiments and wrote the paper with support from Xia Z. Zhao Y performed the theoretical simulations. All authors contributed to the general discussion.

Conflict of interest The authors declare that they have no conflict of interest.

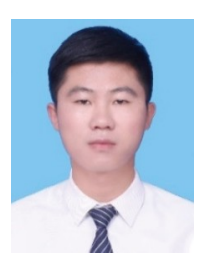

Xinquan Zhou is a $\mathrm{PhD}$ student at the South China University of Technology. He completed his bachelor degree at the University of Jinan in 2017 and received the master degree from Guangdong University of Technology in 2020. His research mainly focuses on the rare-earthions-activated long persistent phosphors and luminescent materials for LEDs.

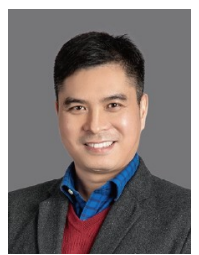

Zhiguo Xia is currently a professor of materials chemistry and physics at the South China University of Technology. He obtained his bachelor degree in 2002 and master degree in 2005 from Beijing Technology and Business University, and he received his $\mathrm{PhD}$ degree from Tsinghua University in 2008. His research interests are in designing of new rare-earth phosphors and luminescent metal halides for emerging photonics applications by integrating structural discovery, modification and structure-property relation studies.

\section{镨掺杂的多响应型深紫外苂光粉与杀菌应用}

周新全 ${ }^{1}$, 乔建伟 ${ }^{1}$, 赵逸飞 ${ }^{1}$, 韩凯 ${ }^{1}$, 夏志国 $1,2^{*}$

摘要 开发能够被不同激发光源激发, 并在同一体系中实现多响应发 射的多模态发光材料是发光材料领域的一个挑战. 本文采用一种异价 掺杂策略, 在富含缺陷的 $\mathrm{Li}_{2} \mathrm{CaGeO}_{4}$ (LCGO)基质中实现了 $\mathrm{Pr}^{3+}$ 掺杂的 多模式深紫外发射. LCGO: $\operatorname{Pr}^{3+}$ 多响应特性使其能够被常见的光源, 包 括X射线管、标准紫外灯、蓝光和近红外激光器激发. $\mathrm{Pr}^{3+}$ 掺杂产生并 重建LCGO材料中的缺陷位点, 包括阳离子缺陷的产生和氧空位的减 少, 进而调控了陷阱分布, 使得该材料同时表现出余辉发光和光激励发 光. 基于 $\mathrm{Pr}^{3+}$ 离子的 $4 f$ 离散能级, 该材料还表现出上转换发光, 入射的可 见光可转化为用于杀菌的深紫外发射. 基于此, 本文设计了一种双模光 转换策略用于灭活细菌. 这种多响应的深紫外发射体为开发用于杀菌 的紫外光源提供了新的思路. 在以陷阱为媒介的基质晶格中实施异价 掺杂或者取代也为构建多模发光材料提供了可行途径. 